\title{
Recurrent Spontaneous Pneumothorax Secondary to Mari- juana-Induced Extensive Bullous Emphysema
}

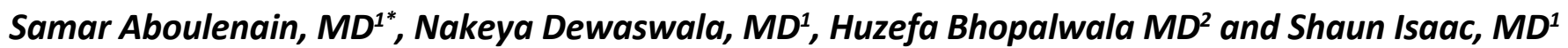

${ }^{1}$ JFK Medical Center, University of Miami, USA

${ }^{2}$ Mayo Clinic Rochester, USA

*Corresponding author: Samar Aboulenain, MD, JFK Medical Center, University of Miami, 180 John F

Kennedy Dr Suite 210, Atlantis, FL 33462, USA, Tel: (561)-531-2655

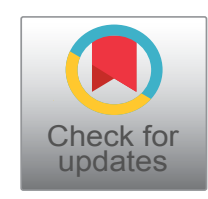

\begin{abstract}
Marijuana is the world's most commonly used illicit drug. Its use is estimated to be around $2 \%$ to $5 \%$ of the worldwide population and the most commonly used route of use is inhalation. Although not completely elucidated, marijuana has been linked with lung damage and bullous formation. We report a case of a young man with a significant history of marijuana smoking who presented with a recurrent large spontaneous pneumothorax refractory to non-surgical management.
\end{abstract}

\section{Introduction}

Marijuana is the most widely used illegal substance around the globe. The use of cannabis is estimated to be between 3 to $5 \%$ of the whole world population [1]. The effect of marijuana smoking on the lungs remains an area of ongoing research. Previous case reports and case series have linked marijuana smoking with lung disease including emphysema, spontaneous pneumothorax and chronic bronchitis [2-5]. We present a case of a young man with a significant history of marijuana smoking who presented with recurrent large spontaneous pneumothorax with a challenging course of management.

\section{Clinical Presentation}

A 39-year-old African American man brought to the emergency department by paramedics after experiencing a sudden-onset sharp right-sided chest pain. The pain started after a coughing spell while smoking marijuana. He also endorsed shortness of breath but denied any physical trauma. His past medical history is significant for a pneumothorax that has resolved three-month ago. Past surgical history and family history were unremarkable. Social history was significant for daily marijuana and tobacco smoking.

Upon presentation, his peripheral capillary oxygen saturation was $96 \%$ on 2 liters by nasal cannula. The remainder of his vital signs were within normal limits. On physical examination, auscultation of the lungs revealed diminished air entry on the right side of the chest.

Hyperresonance was also noted on the right side on chest percussion. Chest radiograph (CXR) revealed large right-sided pneumothorax affecting $50 \%-60 \%$ of the lung (Figure 1). A chest tube was inserted and placed on suction with persistent air leak. A repeat CXR demonstrated an incomplete lung expansion despite continuous drainage. Computed tomography scan (CT) of the chest showed extensive apical bullous emphysema on both sides (Figure 2 and Figure 3). The patient underwent a video-assisted thoracoscopic surgery (VATS) for blebs stapling and pleurodesis.

Post-operative CXR revealed successful re-expansion of the lungs (Figure 4). A nasopharyngeal swab was performed, and COVID-19 PCR testing was negative. The patient was then discharged from the hospital to follow up in the outpatient after smoking cessation counselling.

\section{Discussion}

In this case report we discuss one of the unusual and 


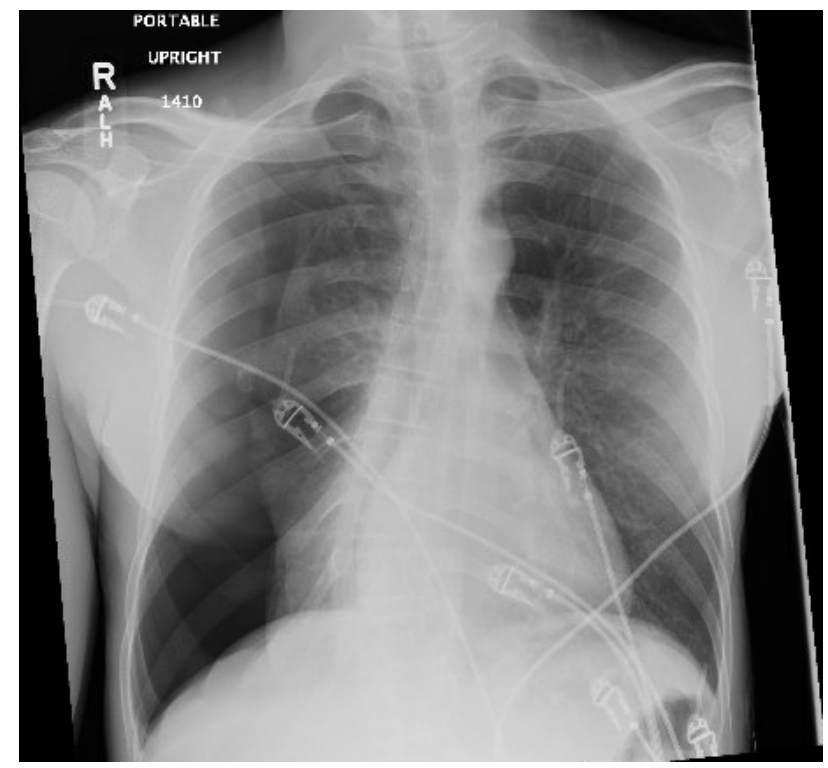

Figure 1: Large right pneumothorax with collapsed right lung.

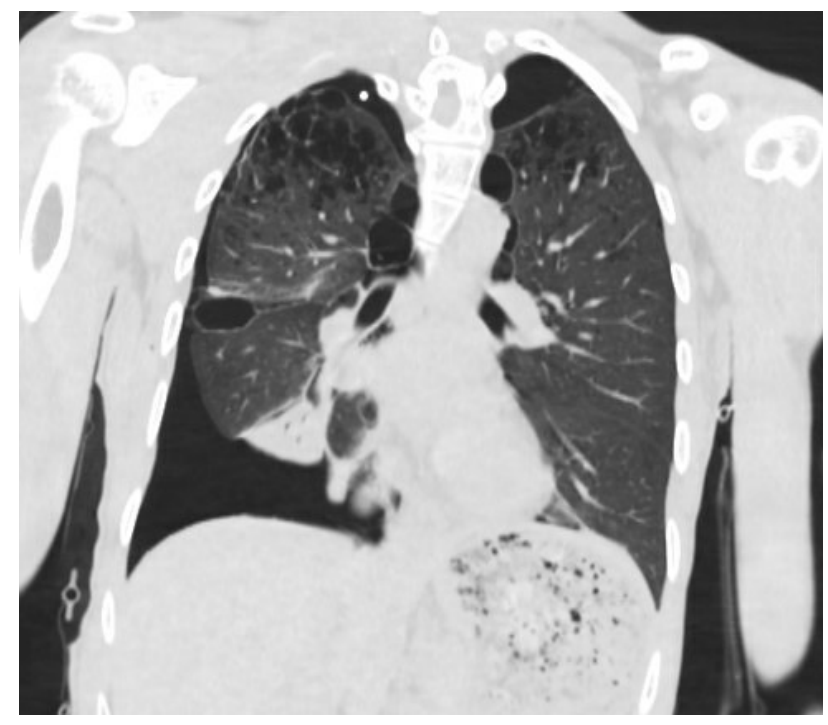

Figure 2: Coronal view of the computed tomography scan shows extensive bilateral apical emphysema, right pneumothorax, and consolidation of the right lung lobe.

life-threatening adverse effects of the most commonly used illicit substance in the United States as well as all around the world. The mechanism of bullae and spontaneous pneumothorax due to marijuana is not yet clear. Helyes, et al. conducted an animal model study that has revealed that marijuana smoking is linked to severe pathophysiological alterations of the lung parenchyma that can yield to emphysema and pneumonitis [6].

Primary spontaneous pneumothoraces usually occur in the absence of lung pathology and is often small in size and benign. However, secondary spontaneous pneumothoraces similar to the presentation of this case is a life-threatening condition and prompt management is critical. Our patient with a history of heavy marijuana and tobacco smoking presented initially with a spontaneous pneumothorax that was drained via thoracosto-

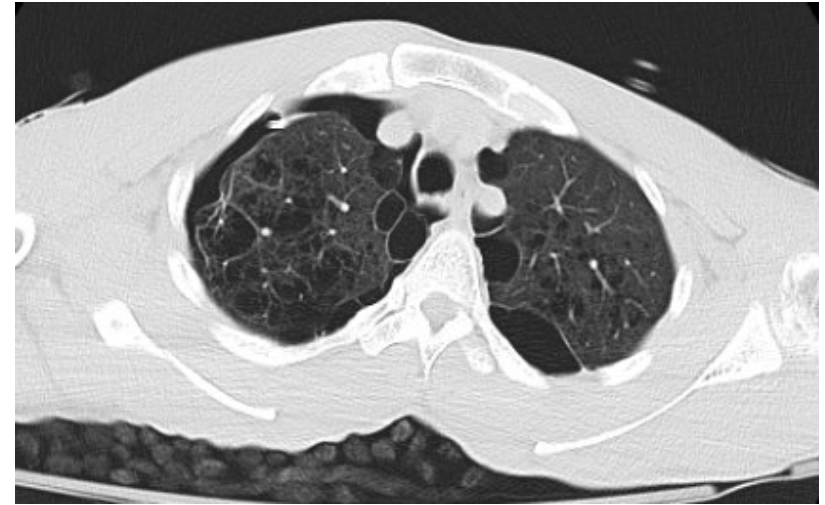

Figure 3: Axial view showing extensive bilateral apical emphysema.

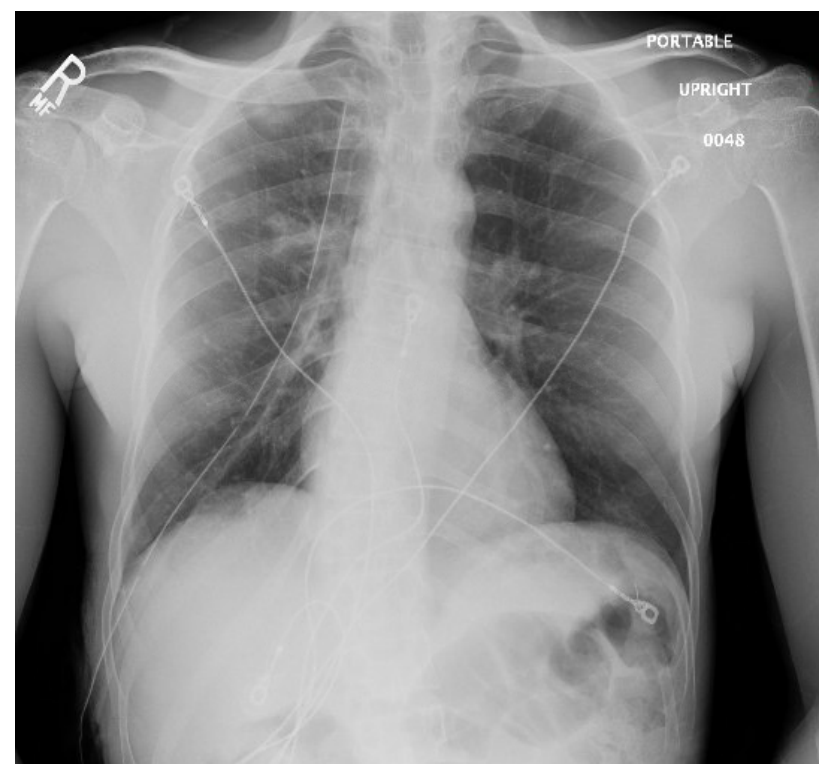

Figure 4: Post-surgical film shows re-expansion of the lung with residual apical pneumothorax and chest tube in place.

my tube and definitive surgical management was postponed due to the coronavirus pandemic. Three months later, he presented with a large recurrent pneumothorax that was potentially life-threatening. His management was complicated with persistent air leak despite prolonged effective drainage requiring surgical intervention for the definite resolution of the pneumothorax. We argue that early surgical management should be considered when encountering a patient with cannabis-induced spontaneous pneumothorax to prevent recurrence and prolonged course of management. A case series by Chardon, et al. has discussed similar presentations of three patients with a history of marijuana abuse associated spontaneous pneumothorax. Among these three patients, two were treated surgically and one was treated conservatively. The patient who was treated conservatively had a more complicated and prolonged course of management.

In our case, the patient continued to smoke cannabis and tobacco after his first episode, increasing his risk of progression of the disease and recurrence of the spon- 
taneous pneumothorax. A retrospective cohort study by Ruppert, et al. found that patients with a history of tobacco smoking were more likely to presentation with spontaneous pneumothorax at a much older age than those with a history of tobacco and cannabis smoking [7].

\section{Conclusion}

Marijuana is not a benign substance and can be linked with lung damage and life-threatening complications. We discussed an unusual case of a young adult with severe bullous emphysema secondary to heavy marijuana use who presented with recurrent large spontaneous pneumothorax refractory to non-surgical management. Surgical intervention should not be delayed in the management of spontaneous pneumothorax secondary to marijuana-induced bullous emphysema to prevent recurrence and reduce the length of hospitalization. Physicians must be aware of the potential pulmonary complication of marijuana to rapidly recognize and promptly manage this condition.

\section{References}

1. Anthony JC, Lopez-Quintero C, Alshaarawy O (2017) Cannabis epidemiology: A selective review. Curr Pharm Des 22: 6340-6352.

2. Underner M, Urban T, Perriot J, Peiffer G, Harika-Germaneau G, et al. (2018) Spontaneous pneumothorax and lung emphysema in cannabis users. Rev Pneumol Clin 74: 400415.

3. Chardon ML, Dalphin JC, Degano B (2018) Pneumothorax in young cannabis smokers: A propos of three cases. Rev Mal Respir 35: 556-561.

4. Figueira Gonçalves JM (2016) Severe bullous emphysema in a young adult cannabis user. Semergen 42: 491-493.

5. Mishra R, Patel R, Khaja M (2017) Cannabis-induced bullous lung disease leading to pneumothorax: Case report and literature review. Medicine 96.

6. Helyes Z, Kemény Á, Csekő K, Szőke E, Elekes K, et al. (2017) Marijuana smoke induces severe pulmonary hyperresponsiveness, inflammation, and emphysema in a predictive mouse model not via CB1 receptor activation. Am J Physiol Lung Cell Mol Physiol 313: 267-277.

7. Ruppert AM, Perrin J, Khalil A, Vieira T, Chedid DA, et al. (2018) Effect of cannabis and tobacco on emphysema in patients with spontaneous pneumothorax. Diagn Interv Imaging 99: 465-471. 